\title{
Obtaining the steady-state drawdown solutions of constant-head and constant-flux tests
}

\author{
Chih-Tse Wang and Hund-Der Yeh* \\ Institute of Environmental Engineering, National Chiao Tung University, Hsinchu, Taiwan
}

\begin{abstract}
:
The solutions of constant-head and constant-flux tests are commonly used to predict the temporal or spatial drawdown distribution or to determine aquifer parameters. Theis and Thiem equations, for instance, are well-known transient and steadystate drawdown solutions, respectively, of the constant-flux test. It is known that the Theis equation is not applicable to the case where the aquifer has a finite boundary or the pumping time tends to infinity. On the other hand, the Thiem equation does not apply to the case where the aquifer boundary is infinite. However, the issue of obtaining the Thiem equation from the transient drawdown solution has not previously been addressed. In this paper, the drawdown solutions for constant-head and constant-flux tests conducted in finite or infinite confined aquifers with or without consideration of the effect of the well radius are examined comprehensively. Mathematical verification and physical interpretation of the solutions to these two tests converging or not converging to the Thiem equation are presented. The result shows that there are some finite-domain solutions for these two tests that can converge to the Thiem equation when the time becomes infinitely large. In addition, the time criteria to give a good approximation to the finite-domain solution by the infinite-domain solution and the Thiem equation are investigated and presented. Copyright () 2008 John Wiley \& Sons, Ltd.
\end{abstract}

KEY WORDS groundwater; aquifer test; Theis equation; Thiem equation; wells

Received 25 June 2007; Accepted 25 October 2007

\section{INTRODUCTION}

The Theis and Thiem equations are the well-known transient and steady-state drawdown solutions, respectively, of a pumping test conducted in confined aquifers. Both solutions are very simple and easily used to predict the aquifer drawdown distribution in practical applications. The Theis equation is derived under the conditions of an infinite extended confined aquifer, neglecting the effect of the well radius. It is important to note that the Theis equation will give an infinite drawdown as the time approaches infinity. Chen (1984) proposed a modified Theis equation for drawdown distribution in a finite confined aquifer and gave a time criterion when applying the Theis equation.

The Thiem equation can be derived either from the continuity equation coupled with Darcy's law (Todd and Mays, 2005) or the radial steady-state flow equation (Charbeneau, 2000). Many researchers have discussed the problem of steady-state flow and warn of erroneous results when applying the Thiem equation to problems of an infinite aquifer (Bear, 1979). Zaadnoordijk (1998) proposed an analytical algorithm based on the superposition principle to simulate transient flow using the Theis equation between any two given steady-state groundwater flows represented by the Thiem equation. His study

\footnotetext{
* Correspondence to: Hund-Der Yeh, Institute of Environmental Engineering, National Chiao Tung University, Hsinchu, Taiwan.

E-mail: hdyeh@mail.nctu.edu.tw
}

implies that the steady-state condition is related to consideration of the effects of the well radius and the finite boundary. However, the issue of obtaining the Thiem equation from the transient drawdown solution has never previously been addressed.

The drawdown distribution may change in response to the constant-head or the constant-flux test. The former holds a specified drawdown in the test well while the latter keeps a constant pumping rate at the pumping well. Mathematically, these two tests can be formulated as different types of boundary value problem. With or without considering the effect of the well radius, many studies have been devoted to developing analytical solutions for these problems under various boundary conditions. The objectives of this current paper are (1) to examine the drawdown solutions obtained by considering or neglecting the effect of the well radius in a finite or infinite confined aquifer for both constant-head and constant-flux tests, (2) to give physical interpretation for the convergences of the transient drawdown solutions of these two tests to the Thiem equation after a long period of aquifer test, and (3) to present criteria for the time when the finite-domain solution can be approximated by the infinite-domain solution or the Thiem equation.

\section{DRAWDOWN SOLUTIONS OF THE CONSTANT-HEAD TEST}

This section considers a constant-head test conducted in a homogeneous and isotropic confined aquifer of constant 
thickness. The one-dimensional radial flow equation describing the drawdown in this confined aquifer can be written as (Batu, 1998)

$$
\frac{\partial^{2} s}{\partial r^{2}}+\frac{1}{r} \frac{\partial s}{\partial r}=\frac{S}{T} \frac{\partial s}{\partial t}
$$

where $s(r, t)$ is the drawdown corresponding to the radial distance $r$ from the test well and the time variable $t, S$ is the storativity, and $T$ is the transmissivity. The drawdown is initially assumed to be zero before the start of the aquifer test, i.e. $s(r, 0)=0$.

For the constant-head test, the drawdown in the test well is maintained constant and denoted $s_{w}$. The rim of the wellbore is selected as the inner boundary and the inner boundary condition for the drawdown can be expressed as $s\left(r_{w}, t\right)=s_{w}$ where $r_{w}$ is the well radius. An outer boundary condition should be provided to solve the flow equation (1). Hereafter, we will present and discuss the solution for the outer boundary specified as a zero drawdown and located at either an infinite or a finite distance from the test well. In general, the zerodrawdown boundary plays a role in supplying water if the head in adjacent area is lower than the zero drawdown. The zero drawdown is often located at the boundary of a water body, such as a river, lake, or reservoir. In addition, the drawdown solution obtained by considering or neglecting the effect of well radius will also be presented.

\section{Infinite domain with a finite well radius}

It is assumed that the outer boundary is located at infinity and the outer boundary condition is expressed as $s(\infty, t)=0$. The solution subject to the infinite outer boundary assumption is referred to as the infinite-domain solution. Using the method of Laplace transforms, the general Laplace-domain solution of (1) can be expressed in terms of bases $I_{0}$ and $K_{0}$, which are modified Bessel functions of the first and second kinds of order zero, respectively. The function $I_{0}$ tends to infinity under the outer boundary condition and therefore must be excluded.

Application of the inverse Laplace transforms under the inner boundary condition yields the drawdown distribution for the constant-head test as (Carslaw and Jaeger, 1959, p.335; Peng et al., 2002)

$$
\begin{aligned}
s(r, t)= & s_{w}\left[1-\frac{2}{\pi} \int_{0}^{\infty} \exp \left(-\frac{T}{S} x^{2} t\right)\right. \\
& \left.\frac{Y_{0}(r x) J_{0}\left(r_{w} x\right)-J_{0}(r x) Y_{0}\left(r_{w} x\right)}{J_{0}^{2}\left(r_{w} x\right)+Y_{0}^{2}\left(r_{w} x\right)} \frac{d x}{x}\right]
\end{aligned}
$$

where $J_{0}$ and $Y_{0}$ are Bessel functions of the first and second kinds of order zero, respectively, and $x$ is a dummy variable.

The flow rate entering the test well can then be obtained by applying Darcy's law to (2). Accordingly, the wellbore flux $Q\left(r_{w}, t\right)$ under the condition that the aquifer has an infinite boundary and a finite well radius is (Peng et al., 2002)

$$
\begin{aligned}
Q\left(r_{w}, t\right)= & \frac{8 T s_{w}}{\pi} \int_{0}^{\infty} \exp \left(-\frac{T}{S} x^{2} t\right) \\
& \frac{1}{J_{0}^{2}\left(r_{w} x\right)+Y_{0}^{2}\left(r_{w} x\right)} \frac{d x}{x}
\end{aligned}
$$

The exponential term on the right-hand side of (2) reduces to zero as time approaches infinity; the steady-state drawdown can thus be obtained as $s_{w}$. However this result does not account for the outer boundary condition $s(\infty, t)=0$. Such a result implies that the steady-state drawdown prevails within the entire domain and at the test well. Also, (3) shows that the wellbore flux decreases with increasing time and approaches zero for large times. This result suggests that under the infinite outer boundary condition there is not enough water to maintain a non-zero flow rate within the aquifer for a constant-head test. Thus (2) does not converge to the Thiem equation at large times.

Peng et al. (2002) proposed an improved algorithm for numerical evaluation of (2) and (3). Their results give dimensionless drawdown and dimensionless wellbore flux in tabular forms with better accuracy than Jaeger and Clarke (1942), Jacob and Lohman (1952), and Jaeger (1956). The solid line in Figure 1 is a plot of dimensionless wellbore flux (3), $Q\left(r_{w}, t\right) /\left(2 \pi T s_{w}\right)$, versus dimensionless time, $T t / r_{w}^{2} S$. It can be observed that the wellbore flux decreases continuously and approaches zero rather than a constant flux as time becomes infinitely large. Similar results were also discussed in Yang and Yeh (2002, Figure 3) when considering the case of singlezone formation, and Zhan and Bian (2006, Figure 5) when decreasing the leakage across an aquitard as the time becomes large.

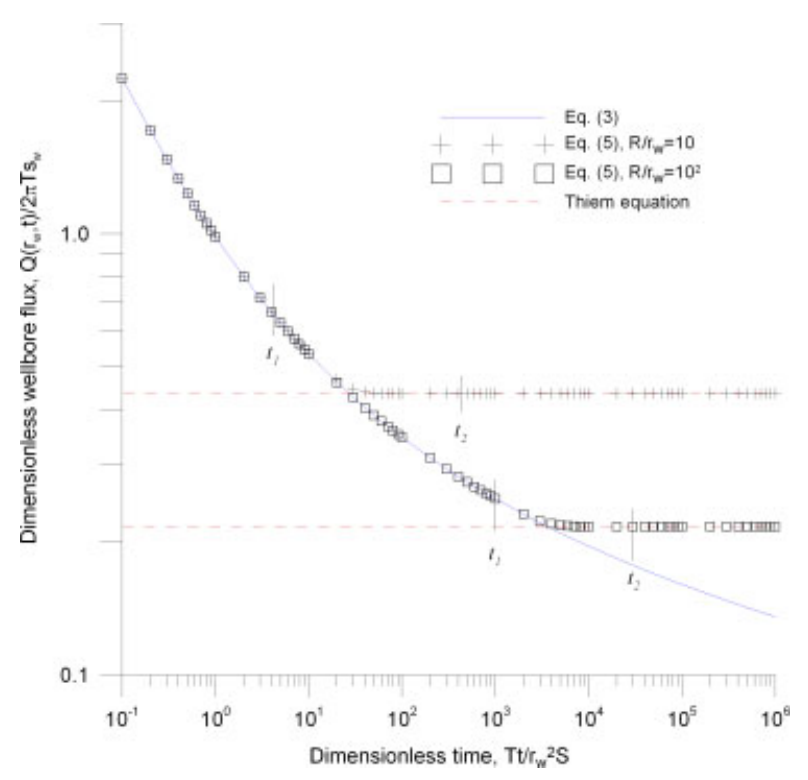

Figure 1. Dimensionless wellbore flux versus dimensionless time for the finite-domain solution (Equation (5)) with $R / r_{w}=10$ and $10^{2}$, the infinite-domain solution (Equation (3)), and the Thiem equation. Note that both Equations (3) and (5) are solved for the constant-head test 


\section{Infinite Domain with Neglecting the Well Radius}

When neglecting the effect of test well radius, that is, $r_{w} \rightarrow 0$, the basis $K_{0}$ of the general Laplace-domain solution tends to infinity and must be excluded. Therefore, there is no solution for this case. The transient and steady-state solutions of the drawdown for the constanthead test with and without considering the effect of well radius under the infinite domain are listed in Table I.

\section{Finite domain with a finite well radius}

In this case, a well of finite radius is considered and a finite distance $R$ from the well is selected to represent the outer boundary where the drawdown is kept at zero. Thus, the solution under a finite outer boundary condition, denoted as $s(R, t)=0$, is referred to as a finite-domain solution. Carslaw and Jaeger (1959, p.332) gave the solution for this problem with Cauchy boundary conditions. By neglecting the flux components of the Cauchy boundary, a drawdown solution of the constanthead test for an aquifer with finite domain and well radius can be obtained as

$$
\begin{aligned}
s(r, t)= & s_{w}\left[\frac{\ln (R / r)}{\ln \left(R / r_{w}\right)}-\pi \sum_{n=1}^{\infty} \exp \left(-\frac{T}{S} \alpha_{n}^{2} t\right)\right. \\
& \left.\frac{J_{0}\left(r_{w} \alpha_{n}\right) Y_{0}\left(r \alpha_{n}\right)-Y_{0}\left(r_{w} \alpha_{n}\right) J_{0}\left(r \alpha_{n}\right)}{\left[J_{0}^{2}\left(r_{w} \alpha_{n}\right)-J_{0}^{2}\left(R \alpha_{n}\right)\right] / J_{0}^{2}\left(R \alpha_{n}\right)}\right]
\end{aligned}
$$

where $\alpha_{n}$ are the roots of $J_{0}\left(r_{w} \alpha\right) Y_{0}(R \alpha)-Y_{0}\left(r_{w} \alpha\right)$ $J_{0}(R \alpha)=0$. A detailed procedure for obtaining (4) is given in Appendix A. The flow rate at wellbore can then be determined as

$$
\begin{aligned}
Q\left(r_{w}, t\right)= & 2 \pi T s_{\mathrm{W}}\left[\frac{1}{\ln \left(R / r_{w}\right)}+2 \sum_{n=1}^{\infty}\right. \\
& \left.\frac{\exp \left(-T \alpha_{n}^{2} t / S\right)}{\left[J_{0}^{2}\left(r_{w} \alpha_{n}\right)-J_{0}^{2}\left(R \alpha_{n}\right)\right] / J_{0}^{2}\left(R \alpha_{n}\right)}\right]
\end{aligned}
$$

Obviously, the steady-state wellbore flux of (5) for time approaching infinity is $Q\left(r_{w}\right)=2 \pi T s_{w} / \ln \left(R / r_{w}\right)$ which indeed is the Thiem equation. Figure 1 shows that the curve (5), i.e. the finite-domain solution, coincides with (3), i.e. the infinite-domain solution, at early stages of the constant-head test and asymptotically approaches the Thiem equation after a long period of time. In other words, the infinite-domain solution can approximate the finite-domain solution when time is less than the boundary-effect time criterion $t_{1}$, implying that the finite boundary has no effect on the wellbore flux. In addition, the finite-domain solution can be reduced to the Thiem equation when time is greater than steadystate time criterion $t_{2}$, implying that the wellbore flux can be considered at steady state. It is worth noting that both the Thiem equation and the infinite-domain solution have the advantage of computing the drawdown solution more easily than the finite-domain solution.

Figure 1 indicates that both dimensionless time criteria $T t_{1} / r_{w}^{2} S$ and $T t_{2} / r_{w}^{2} S$ increase with the dimensionless distance $R / r_{w}$. The values of $T t_{1} / r_{w}^{2} S$ and $T t_{2} / r_{w}^{2} S$, for instance, are 4 and $4 \times 10^{2}$ for $R / r_{w}=10$ and $1 \times 10^{3}$ and $3 \times 10^{4}$ for $R / r_{w}=10^{2}$, respectively, if one defines that the absolute difference in dimensionless wellbore flux between (3) and (5) is less than $10^{-5}$. Thus, the time criterion for $t_{1}$ can be obtained approximately as $R^{2} S / 10 T$ based on a linear relationship between $T t_{1} / r_{w}^{2} S$ and $R / r_{w}$ for the constant-head test. Using the same approach, $t_{2}$ is $4 R^{2} S / T$ approximately. Table II gives a list of time criteria for the finite-domain solution.

\section{Finite domain with neglecting the well radius}

In this case, the outer boundary of the radial flow system is located at a finite distance from the test well and the inner boundary of the well radius is negligible. Note that the general Laplace-domain solution for drawdown in a constant-head test contains the bases of $I_{0}$ and

Table II. The boundary-effect and steady-state time criteria for finite-domain solutions

\begin{tabular}{|c|c|c|c|c|}
\hline \multirow[t]{2}{*}{ State } & \multicolumn{2}{|c|}{ Constant-head test } & \multicolumn{2}{|c|}{ Constant-flux test } \\
\hline & Infinite domain & Finite domain & Infinite domain & Finite domain \\
\hline $\begin{array}{l}\text { Transient } \\
\text { Steady }\end{array}$ & $\underset{*}{\text { Equation }} \underset{*}{(2)}$ & $\begin{array}{l}\text { Considering well radius } \\
\text { Equation (4) } \\
\text { Thiem equation }\end{array}$ & $\underset{*}{\text { Equation }}(8)$ & $\begin{array}{l}\text { Equation (11) } \\
\text { Thiem equation }\end{array}$ \\
\hline $\begin{array}{l}\text { Transient } \\
\text { Steady }\end{array}$ & $\begin{array}{l}* \\
*\end{array}$ & $\begin{array}{c}\text { Neglecting well radius } \\
* \\
*\end{array}$ & $\begin{array}{c}\text { Theis equation } \\
{ }_{*}\end{array}$ & $\begin{array}{l}\text { Equation (12) } \\
\text { Thiem equation }\end{array}$ \\
\hline
\end{tabular}

\begin{tabular}{lcc}
\hline Solution type & $\begin{array}{c}\text { Boundary-effect time } \\
\text { criterion, } t_{1}\end{array}$ & $\begin{array}{c}\text { Steady-state time } \\
\text { criterion, } t_{2}\end{array}$ \\
\hline Constant-head test & $\frac{1}{10} \tau$ & $4 \tau$ \\
Constant-flux test & $\frac{3}{100} \tau$ & $8 \tau$ \\
$\begin{array}{l}\text { Constant-flux test } \\
\text { when neglecting } \\
\text { well radius }\end{array}$ & $\frac{1}{16} \tau$ & $\frac{10^{5}}{4} \tau$ \\
\hline
\end{tabular}

$\tau=R^{2} S / T$

Table I. Transient and steady-state drawdown solutions of constant-head and constant flux tests

\footnotetext{
* Indicates that the solution does not exit.
} 
$K_{0}$. The basis $K_{0}$ has to be excluded from the general solution as $r_{w} \rightarrow 0$ and the remaining basis $I_{0}$ cannot satisfy the boundary conditions of fixed drawdown at wellbore and zero drawdown at the finite outer boundary simultaneously. Consequently, there is no solution for this case. Table I also lists the transient and steady-state drawdown solutions for the constant-head test when the outer boundary is finite, with and without considering the effect of well radius.

Interestingly, a heat problem presented in Carslaw and Jaeger $(1959$, p.327) is similar to the case mentioned above but admits a solution. Analogous to the groundwater flow, their inner boundary condition has a finite drawdown instead of fixed drawdown and the outer boundary condition at a finite distance $R$ from the test well has a fixed drawdown rather than zero drawdown. The solution can be written as (Carslaw and Jaeger, 1959, p.328, Equation (7))

$$
s(r, t)=s_{R}-\frac{2 s_{R}}{R} \sum_{n=1}^{\infty} \exp \left(-\frac{T}{S} \theta_{n}^{2} t\right) \frac{J_{0}\left(r \theta_{n}\right)}{\theta_{n} J_{1}\left(R \theta_{n}\right)}
$$

where $s_{R}$ represents a fixed drawdown at finite distance $R, \theta_{n}$ are the roots of $J_{0}(R \theta)=0$ and $J_{1}$ is the Bessel function of the first kind of order one. This solution, (6), is extractable due to the fact that the inner boundary condition is finite and allowed to vary instead of being fixed in the constant-head test.

\section{DRAWDOWN SOLUTIONS OF THE CONSTANT-FLUX TEST}

This section considers a constant-flux test conducted at a confined aquifer. The initial condition for drawdown before the start of the pumping test is assumed to be zero. The drawdown solutions for (1) are presented for the cases that the outer boundary specified as zero drawdown is located at either an infinite or a finite domain and the inner boundary considers or does not consider the effect of well radius.

\section{Infinite domain with finite well radius}

The constant-flux test has a constant pumping rate $Q_{p}$. Thus, the inner boundary condition for the constant-flux test may be written as

$$
\left.2 \pi r_{w} T \frac{\partial s}{\partial r}\right|_{r=r_{w}}=-Q_{p}
$$

The drawdown solution of the constant-flux test subject to (7) and zero drawdown at infinity can be obtained by Laplace transforms as (Carslaw and Jaeger, 1959, p.338)

$$
\begin{aligned}
s(r, t)= & \frac{Q_{p}}{\pi^{2} r_{w} T} \int_{0}^{\infty}\left[1-\exp \left(-\frac{T}{S} x^{2} t\right)\right] \\
& \frac{Y_{0}(r x) J_{1}\left(r_{w} x\right)-J_{0}(r x) Y_{1}\left(r_{w} x\right)}{J_{1}^{2}\left(r_{w} x\right)+Y_{1}^{2}\left(r_{w} x\right)} \frac{d x}{x^{2}}
\end{aligned}
$$

where $Y_{1}$ is the Bessel function of the second kind of order one. Papadopulos and Cooper (1967) also developed a drawdown solution to the constant-flux test by further considering the wellbore storage, and their inner boundary can be expressed as

$$
\left.2 \pi r_{w} T \frac{\partial s}{\partial r}\right|_{r=r_{w}}-\pi r_{w}^{2} \frac{d H}{d t}=-Q_{p}
$$

where $H(t)$ is the well water level. Therefore, their drawdown solution can be reduced to (8) if one ignores the wellbore storage in (9). In addition, Cooper et al. (1967) also gave a well water level solution of the slug test by considering the wellbore storage.

Yeh et al. (2003) presented a closed-form solution with detailed numerical evaluations for a radial two-zone drawdown equation for groundwater under constant-flux pumping in a finite-radius well. The evaluation of (8) can be obtained if the transmissivities and storativities for the skin zone and formation zone are the same as in their article.

Figure 2 gives a graphical representation of dimensionless drawdown of (8) for dimensionless distance $r / r_{w}=$ $10^{2}$ and dimensionless time $T t /\left(r_{w}^{2} S\right)$ ranging from $10^{2}$ to $10^{9}$. The plot of drawdown distribution demonstrates that drawdown increases with time and becomes infinity as time approaches infinity. This implies that the outer boundary cannot provide enough water to balance continuous well pumping and the aquifer is overdrawn as time becomes infinitely large. This result indicates that the transient drawdown solution of the constant-flux test when considering the effects of well radius and the infinite domain does not reduce to the Thiem equation at very large times.

\section{Infinite domain, neglecting well radius}

The solution of the constant-flux test conducted in an infinite domain obtained by neglecting the effect of

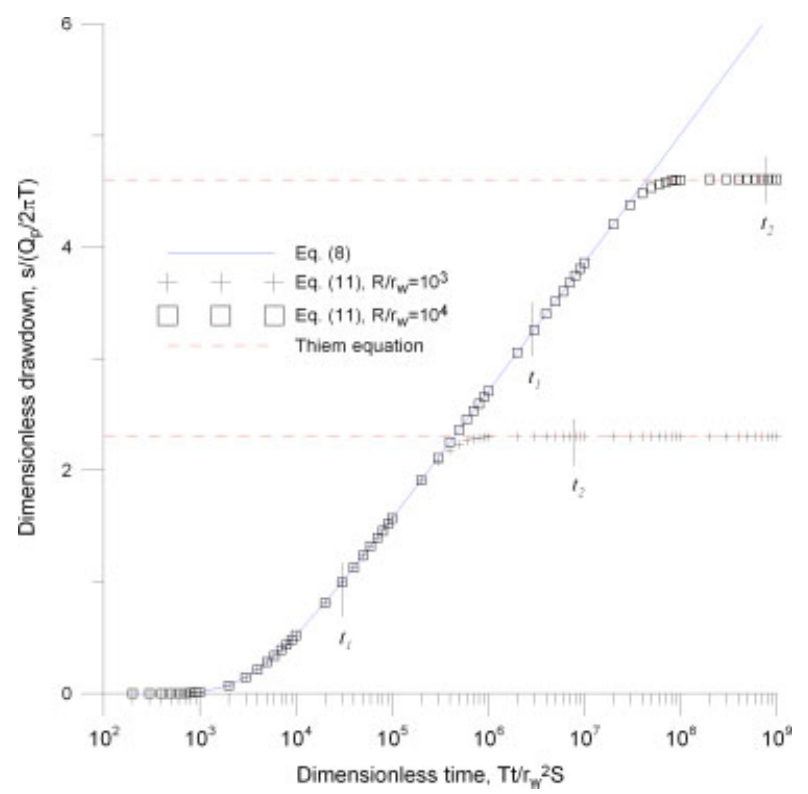

Figure 2. Dimensionless drawdown versus dimensionless time for the finite-domain solution (Equation (11)) with $R / r_{w}=10^{3}$ and $10^{4}$ and $r / r_{w}=10^{2}$, the infinite-domain solution (Equation (8)), and the Thiem equation. Note that both Equations (8) and (11) are solved for the constant-flux test 
well radius is analysed in this case. By applying the Laplace transforms and the asymptotic form of modified Bessel function (Abramowitz and Stegun, 1979, p.375), one obtains the drawdown equation as

$$
s(r, t)=\frac{Q_{p}}{4 \pi T} \int_{u}^{\infty} \frac{\exp (-x)}{x} d x
$$

where $u=r^{2} S /(4 T t)$, indicating that the value of $u$ is inversely proportional to time.

Equation (10) is the well-known Theis equation and the integral in (10) is called the well function and expressed as $W(u)$. This function tends to infinity when time approaches infinity and/or radial distance approaches zero; thus the drawdown of the Theis equation also becomes infinite. In addition, Cooper-Jacob's equation, a special case of the Theis equation under the condition $u<0.01$, should also give infinite drawdown when $u$ approaches zero. Note that the expression for the difference in the drawdowns at any two points within the aquifer is the same as the Thiem equation if the drawdown is expressed in terms of Cooper-Jacob's equation. However, this expression is applicable only when the values of $u$ for both points are less than 0.01 . Accordingly, the locations of these two points should not be too far away from the pumping well and the expression for the drawdown difference based on Cooper-Jacob's equation does not apply to the entire aquifer domain.

The transient and steady-state drawdown solutions of a constant-flux test conducted in an aquifer with an infinite outer boundary are compared in Table I. The results for the constant-head and constant-flux tests indicate that these two tests in an infinite domain do not have steadystate solutions; thus, (2), (8), and the Theis equation cannot reduce to the Thiem equation.

\section{Finite domain with finite well radius}

Similar to the development of (4), the drawdown solution for (1) subject to (7) and the condition of zero drawdown at a finite domain can be obtained as, according to Carslaw and Jaeger (1959, p.334),

$$
\begin{aligned}
s(r, t)= & \frac{Q_{p}}{2 \pi T}\left[\ln \frac{R}{r}-\frac{\pi}{r_{w}} \sum_{n=1}^{\infty} \exp \left(-\frac{T}{S} \beta_{n}^{2} t\right)\right. \\
& \left.\frac{J_{1}\left(r_{w} \beta_{n}\right) Y_{0}\left(r \beta_{n}\right)-Y_{1}\left(r_{w} \beta_{n}\right) J_{0}\left(r \beta_{n}\right)}{\beta_{n}\left[J_{1}^{2}\left(r_{w} \beta_{n}\right)-J_{0}^{2}\left(R \beta_{n}\right)\right] / J_{0}^{2}\left(R \beta_{n}\right)}\right]
\end{aligned}
$$

where $\beta_{n}$ represent the roots of $J_{1}\left(r_{w} \beta\right) Y_{0}(R \beta)$ $-Y_{1}\left(r_{w} \beta\right) J_{0}(R \beta)=0$. It is noteworthy that the numerator of the second term in brackets of (11) equals $2 /\left(\pi r_{w} \beta_{n}\right)$ when $r=r_{w}$ and the related drawdown solution was given in van Everdingen and Hurst (1949). The steady-state drawdown solution can easily be obtained by setting the time to infinity in (11), which is indeed the Thiem equation if the distance $r$ is equal to the well radius.

Figure 2 shows the distribution of dimensionless drawdown of (11) versus dimensionless time for $R / r_{w}$ being equal to $10^{3}$ and $10^{4}$ with $r / r_{w}=10^{2}$. It also demonstrates that the curve of (11), i.e. finite-domain solution, can be approximated by (8), i.e. infinite-domain solution, at early times and reduces to the Thiem equation after a long period of time. The former indicates the drawdown distribution that behaves as in an infinite aquifer before having the boundary effect. The latter implies that the drawdown distribution almost reaches its steady-state condition and the Thiem equation is applicable if the time is larger than the steady-state time criterion. Similar to the analysis used in the constant-head test, $t_{1}$ is obtained as $3 R^{2} S / 100 T$ approximately for the constant-flux test when considering the well radius if the absolute difference of dimensionless drawdown between (11) and (8) is less than $10^{-5}$. In addition, $t_{2}$ approximately equals $8 R^{2} S / T$ if the absolute difference of dimensionless drawdown between (11) and Thiem equation is also less than $10^{-5}$.

\section{Finite domain, neglecting well radius}

In this case, we consider that the outer boundary is located at some finite distance and the well radius effect is negligible. Chen (1984) gave the drawdown solution as

$$
s(r, t)=\frac{Q_{p}}{4 \pi T}[W(u)-W(U)+2 \Phi]
$$

and

$$
\begin{aligned}
\Phi= & \sum_{n=1}^{\infty} \frac{J_{0}\left\lfloor(u / U)^{1 / 2} \chi_{n}\right\rfloor}{\chi_{n} J_{1}\left(\chi_{n}\right)} \cdot \int_{0}^{1} \exp \\
& {\left[\frac{-U}{x}-\frac{\chi_{n}^{2}(1-x)}{4 U}\right] \frac{d x}{x} }
\end{aligned}
$$

where $U$ is $R^{2} S /(4 T t)$ and $\chi_{n}$ represents the roots of Bessel function $J_{0}\left(\chi_{n}\right)=0$.

Both the arguments $u$ and $U$ in (12) and (13) are inversely proportional to time. The functions $W(u)$ and $W(U)$ can be expressed in terms of an infinite series and approximated as $-(0.5772+\ln u)$ and $-(0.5772+$ $\ln U$ ), respectively, as $u \rightarrow 0$ and $U \rightarrow 0$ after a long period of time (Todd and Mays, 2005). The difference of $W(u)$ and $W(U)$ reduces to $2 \ln (R / r)$ where $r$ should not be equal to zero. Moreover, the exponential term in the integrand of (13) approaches zero as $U \rightarrow 0$ and the value of $\Phi$ is negligible. As a result, the drawdown of (12) approaches the Thiem equation when time approaches infinity. The results of transient and steadystate drawdown solutions for the constant-flux test with a finite outer boundary when considering or neglecting well radius effects are also shown in Table I.

Again, Equation (12), i.e. a finite-domain solution, can be approximated by the Theis equation (10) when $U \geq 4$ (Chen, 1984) and reduces to the Thiem equation when $U \leq 10^{-5}$ if the absolute difference of dimensionless drawdown is less than $10^{-5}$. The time criteria $t_{1}$ and $t_{2}$ for the constant-flux test when neglecting the well radius effect are obtained as $R^{2} S / 16 T$ and $10^{5} R^{2} S / 4 T$, respectively, as shown in Table II. 


\section{CONCLUDING REMARKS}

This paper addresses the development of steady-state solutions from transient drawdown solutions of constanthead and constant-flux tests in a finite or infinite domain, with or without considering the well radius. The results show that the finite domain condition is sufficient for the development of a steady-state solution from a transient drawdown solution. Such a condition ensures that the mass balance between extraction and supply is satisfied and the flow can reach its steady state condition within a finite domain. In addition, time criteria, which may be useful in practical applications, are provided for the approximation of the finite-domain solution by the infinite-domain solution or Thiem equation. It is found that the infinite-domain solutions can be used to determine the drawdown distribution or the aquifer parameters if the time is smaller than the boundary-effect time criterion for an aquifer with a finite domain. Similarly, the Thiem equation is applicable whenever the time is larger than the steady-state time criterion.

\section{ACKNOWLEDGEMENT}

Research leading to this paper has been partially supported by the grants from Taiwan National Science Council under contract number NSC 95-2221-E-009-017. The authors sincerely thank two anonymous reviewers for constructive comments and suggested revisions.

\section{REFERENCES}

Abramowitz M, Stegun IA. 1979. Handbook of Mathematical Functions. Dover Publications: New York.

Batu V. 1998. Aquifer Hydraulics. John Wiley: New York.

Bear J. 1979. Hydraulics of Groundwater. McGraw-Hill: New York.

Carslaw HS, Jaeger JC. 1959. Conduction of Heat in Solids, 2nd edn. Oxford University Press: London.

Charbeneau RJ. 2000. Groundwater Hydraulics and Pollutant Transport. Prentice Hall: New York.

Chen CS. 1984. A reinvestigation of the analytical solution for drawdown distributions in a finite confined aquifer. Water Resources Research 20: $1466-1468$

Cooper Jr HH, Bredehoeft JD, Papadopulos IS. 1967. Response of a finite-diameter well to an instantaneous charge of water. Water Resources Research 3(1): 263-269.

Jacob CE, Lohman SW. 1952. Nonsteady flow to a well of constant drawdown in an extensive aquifer. Transactions, American Geophysical Union 33(4): 559-569.

Jaeger JC. 1956. Numerical values for the temperature in radial heat flow. Journal of Mathematical Physics 34: 316-321.

Jaeger JC, Clarke M. 1942. A short table of $\mathrm{I}(0, \mathrm{i} ; \mathrm{x})$. Proceedings of the Royal Society of Edinburgh, Section A 61: 229-230.

Papadopulos IS, Cooper Jr HH. 1967. Drawdown in a well of large diameter. Water Resources Research 3(1): 241-244.

Peng HY, Yeh HD, Yang SY. 2002. Improved numerical evaluation for the radial groundwater flow equation. Advances in Water Resources 25 : $663-675$.

Todd DK, Mays LM. 2005. Groundwater Hydrology. John Wiley: New York.

van Everdingen AF, Hurst W. 1949. The application of the Laplace transformation to flow problems in reservoirs. Petroleum Transactions, AIME 186: 305-324.

Yang SY, Yeh HD. 2002. Solution for flow rates across the wellbore in a two-zone confined aquifer. Journal of Hydraulic Engineering 128: 175-183. DOI: 10·1061/(ASCE)0733-9429(2002)128:2(175).
Yeh HD, Yang SY, Peng HY. 2003. A new closed-form solution for a radial two-layer drawdown equation for groundwater under constantflux pumping in a finite-radius well. Advances in Water Resources 26: 747-757.

Zaadnoordijk WJ. 1998. Transition from transient Theis wells to steady Thiem wells. Hydrological Sciences Journal 43: 859-873.

Zhan H, Bian A. 2006. A method of calculating pumping induced leakage. Journal of Hydrology 328: 659-667. DOI: 10·1016/j.jhydrol.2006·01·010.

\section{APPENDIX A: DERIVATION OF EQUATION}

General solutions of the one-dimensional radial heat conduction equation, which is analogous to the groundwater drawdown equation, subject to Cauchy boundary conditions at the edges of a hollow cylinder were given in Carslaw and Jaeger (1959, p.332). The Cauchy boundary conditions in terms of drawdown were expressed as

$$
k_{1} \frac{\partial s}{\partial r}-k_{2} s=k_{3}, \quad r=a
$$

and

$$
k_{1}^{\prime} \frac{\partial s}{\partial r}-k_{2}^{\prime} s=k_{3}^{\prime}, \quad r=b
$$

where $k_{1}, k_{2}, k_{3}, k_{1}^{\prime}, k_{2}^{\prime}, k_{3}^{\prime}$ are constant; $a$ and $b$ are the radial distance of the inner boundary and outer boundary in the considered region, respectively. Equations (A1) and (A2) represent the combination of the constant-head and constant-flux boundary conditions.

Using Laplace transforms, the drawdown solution based on (A1) and (A2) is

$$
\begin{aligned}
& s(r, t)=A_{1}-\pi \sum_{n=1}^{\infty} \exp \left(-\frac{T}{S} \alpha_{n}^{2} t\right) \\
& \frac{\left\lfloor k_{1}^{\prime} \alpha_{n} J_{1}\left(b \alpha_{n}\right)-k_{2}^{\prime} J_{0}\left(b \alpha_{n}\right)\right\rfloor C_{0}\left(r, \alpha_{n}\right)}{F\left(\alpha_{n}\right)} A_{2}\left(\alpha_{n}\right)
\end{aligned}
$$

where

$$
\begin{aligned}
& A_{1}=\frac{-a k_{3}\left\lfloor k_{1}^{\prime}-b k_{2}^{\prime} \ln (r / b)\right\rfloor+b k_{3}^{\prime}\left[k_{1}+a k_{2} \ln (r / a)\right]}{a k_{2} k_{1}^{\prime}+b k_{1} k_{2}^{\prime}+a b k_{2} k_{2}^{\prime} \ln (b / a)} \\
& A_{2}=k_{3}\left\lfloor k_{1}^{\prime} \alpha_{n} J_{1}\left(b \alpha_{n}\right)-k_{2}^{\prime} J_{0}\left(b \alpha_{n}\right)\right\rfloor \\
& -k_{3}^{\prime}\left[k_{1} \alpha_{n} J_{1}\left(a \alpha_{n}\right)+k_{2} J_{0}\left(a \alpha_{n}\right)\right] \\
& C_{0}\left(r, \alpha_{n}\right)=J_{0}\left(r \alpha_{n}\right)\left[k_{1} \alpha_{n} Y_{1}\left(a \alpha_{n}\right)+k_{2} Y_{0}\left(a \alpha_{n}\right)\right] \\
& -Y_{0}\left(r \alpha_{n}\right)\left[k_{1} \alpha_{n} J_{1}\left(a \alpha_{n}\right)+k_{2} J_{0}\left(a \alpha_{n}\right)\right] \\
& F\left(\alpha_{n}\right)=\left(k_{1}^{\prime 2} \alpha_{n}^{2}+k_{2}^{\prime 2}\right)\left[k_{1} \alpha_{n} J_{1}\left(a \alpha_{n}\right)+k_{2} J_{0}\left(a \alpha_{n}\right)\right]^{2} \\
& -\left(k_{1}^{2} \alpha_{n}^{2}+k_{2}^{2}\right)\left[k_{1}^{\prime} \alpha_{n} J_{1}\left(b \alpha_{n}\right)-k_{2}^{\prime} J_{0}\left(b \alpha_{n}\right)\right]^{2}
\end{aligned}
$$

and $\alpha_{\mathrm{n}}$ are the positive roots of

$$
\begin{aligned}
& {\left[k_{1} \alpha J_{1}(a \alpha)+k_{2} J_{0}(a \alpha)\right]\left\lfloor k_{1}^{\prime} \alpha Y_{1}(b \alpha)-k_{2}^{\prime} Y_{0}(b \alpha)\right\rfloor} \\
& \quad-\left[k_{1} \alpha Y_{1}(a \alpha)+k_{2} Y_{0}(a \alpha)\right]\left[k_{1}^{\prime} \alpha J_{1}(b \alpha)-k_{2}^{\prime} J_{0}(b \alpha)\right]=0
\end{aligned}
$$

For the constant-head test with a finite well radius and outer boundary, the constants in (A1) and (A2) can be replaced by $k_{1}=0, k_{2}=-1, k_{3}=s_{w}, k_{1}^{\prime}=0, k_{2}^{\prime}=1$, $k_{3}^{\prime}=0, a=r_{w}$, and $b=R$. By careful substitution, the drawdown (4) can be obtained from (A3). 